\title{
A metamaterial having a frequency dependent elasticity tensor and a zero effective mass density
}

\author{
Graeme Milton \\ Department of Mathematics, University of Utah, Salt Lake City UT 84112, USA \\ Pierre Seppecher \\ Institut de Mathématiques de Toulon \\ Université de Toulon et du Var, BP 132-83957 La Garde Cedex, France
}

\begin{abstract}
Within the context of linear elasticity we show that a two-terminal network of springs and masses, can respond exactly the same as a normal spring, but with a frequency dependent spring constant. As a consequence a network of such springs can have a frequency dependent effective elasticity tensor but zero effective mass density. The internal masses influence the elasticity tensor, but do not contribute to the effective mass density at any frequency.
\end{abstract}

Keywords: metamaterials, effective mass density.

\section{Introduction}

The mass density term which enters the macroscopic wave equation of linear continuum elastodynamics need not be the same as the mass density calculated by local volume averaging the interior masses. This was observed by Berryman [1] in the context of an approximation scheme for suspensions of particles in an inviscid fluid and later found to be more generally true for composites constructed from components having a high contrast in stiffness and density. The reason is simply that interior masses do not necessarily locally move together in lock step motion with each other, even when the wavelength is long, and therefore the locally averaged momentum is not simply a product of the locally averaged mass times the locally averaged velocity [2]. At a given frequency, the effective mass density entering the wave equation can be anisotropic, negative, or even complex [3, 4, 5, 6, 7, 2, 8. In general, the vibrations of interior masses cause the effective elasticity tensor and the effective mass density to depend on frequency. This raises the interesting question, which will be explored here, as to whether internal masses can influence the effective elasticity tensor, and cause it to be frequency dependent, but not contribute to the overall effective mass density at any frequency? We will see the answer for mass-spring networks is yes, theoretically it can, provided we are strictly working within the framework of linear elasticity and ignore gravity and non-linear instabilities such as buckling. 


\section{Dispersive Normal Springs}

If we have a normal spring with spring constant $k$ and terminals at the points $\mathbf{x}_{1}$ and $\mathbf{x}_{2}$ then the response of the spring, relating the two forces $\mathbf{f}_{1}$ and $\mathbf{f}_{2}$ applied to the spring at the terminals to the displacements $\mathbf{u}_{1}$ and $\mathbf{u}_{2}$ there, takes the form

$$
\left[\begin{array}{l}
\mathbf{f}_{1} \\
\mathbf{f}_{2}
\end{array}\right]=k \mathbf{n} \cdot\left(\mathbf{u}_{1}-\mathbf{u}_{2}\right)\left[\begin{array}{c}
\mathbf{n} \\
-\mathbf{n}
\end{array}\right], \quad \text { where } \mathbf{n}=\frac{\mathbf{x}_{1}-\mathbf{x}_{2}}{\left|\mathbf{x}_{1}-\mathbf{x}_{2}\right|}
$$

We will define a dispersive normal spring to be any two terminal network of springs and masses such that the relation between the forces and displacements at the terminals at any frequency takes the form (1), but with $k$ being dependent on frequency. In particular, the relation (11) implies that the forces $\mathbf{f}_{1}$ and $\mathbf{f}_{2}$ are equal in magnitude and opposite in direction, and directed parallel to $\mathbf{x}_{1}-\mathbf{x}_{2}$.

An essentially explicit scheme for constructing dispersive normal springs is a corollary of Theorem 4 of Guevara Vasquez, Milton and Onofrei [9]. That theorem covers the much more general case of characterizing all possible time-dependent responses of multiterminal mass-spring networks, and generalized earlier work of Camar-Eddine and Seppecher [10] on characterizing the response of multiterminal spring networks. Our objective here is to present a simpler construction where the mechanism responsible for the behavior of the network is easy to grasp.

To construct a dispersive normal spring, consider first the four terminal spring network of figure 1, consisting of a square diamond of identical springs with four other identical springs as legs, which extend directly outwards from the four vertices of the diamond to the terminals. This network is a rank one network: it only supports one loading (and all multiples of this loading). To see this suppose the spring linking terminal 1 to the diamond is under tension $T$. Then, by balance of forces, the four springs in the diamond must be under tension $T / \sqrt{2}$ and the other legs joining the diamond to the terminal edges must be under tension $T$. Thus forces at all the four terminal nodes are determined if we know the force at one terminal node : the response of this network, relating the four forces $\mathbf{f}_{1}, \mathbf{f}_{2}, \mathbf{f}_{3}$ and $\mathbf{f}_{4}$ at the terminals to the displacements $\mathbf{u}_{1}, \mathbf{u}_{2}, \mathbf{u}_{3}$ and $\mathbf{u}_{4}$ there necessarily takes the form $\left(\mathbf{f}_{1}, \mathbf{f}_{2}, \mathbf{f}_{3}, \mathbf{f}_{4}\right)=\left(T \mathbf{n},-T \mathbf{n}, T \mathbf{n}_{\perp},-T \mathbf{n}_{\perp}\right)$ where the scalar tension $T$ depends linearly on $\mathbf{u}_{1}, \mathbf{u}_{2}, \mathbf{u}_{3}$ and $\mathbf{u}_{4}$ (here $\mathbf{n}$ denotes the unit vector pointing from $\mathbf{x}_{2}$ to $\mathbf{x}_{1}$ and $\mathbf{n}_{\perp}$ is the orthogonal unit vector pointing from $\mathbf{x}_{4}$ to $\mathbf{x}_{3}$ ). Thus the response matrix $\mathbf{W}$ which relates the forces at the terminals to the displacements there, via the linear relation,

$$
\left[\begin{array}{l}
\mathbf{f}_{1} \\
\mathbf{f}_{2} \\
\mathbf{f}_{3} \\
\mathbf{f}_{4}
\end{array}\right]=\mathbf{W}\left[\begin{array}{l}
\mathbf{u}_{1} \\
\mathbf{u}_{2} \\
\mathbf{u}_{3} \\
\mathbf{u}_{4}
\end{array}\right]
$$

is a rank-one matrix. Since (no matter what the network), this response matrix is a positive semidefinite symmetric matrix, we deduce that

$$
\left[\begin{array}{l}
\mathbf{f}_{1} \\
\mathbf{f}_{2} \\
\mathbf{f}_{3} \\
\mathbf{f}_{4}
\end{array}\right]=g\left[\begin{array}{c}
\mathbf{n} \\
-\mathbf{n} \\
\mathbf{n}_{\perp} \\
-\mathbf{n}_{\perp}
\end{array}\right]\left[\begin{array}{llll}
\mathbf{n}^{T}-\mathbf{n}^{T} & \mathbf{n}_{\perp}^{T}-\mathbf{n}_{\perp}^{T}
\end{array}\right]\left[\begin{array}{l}
\mathbf{u}_{1} \\
\mathbf{u}_{2} \\
\mathbf{u}_{3} \\
\mathbf{u}_{4}
\end{array}\right]=g\left(\mathbf{n} \cdot\left(\mathbf{u}_{1}-\mathbf{u}_{2}\right)+\mathbf{n}_{\perp} \cdot\left(\mathbf{u}_{3}-\mathbf{u}_{4}\right)\right)\left[\begin{array}{c}
\mathbf{n} \\
-\mathbf{n} \\
\mathbf{n}_{\perp} \\
-\mathbf{n}_{\perp}
\end{array}\right]
$$


where the constant $g>0$ scales in proportion to the stiffness of the springs in the network. Now, let us place a mass $m$ at the terminals 3 and 4 , and make them interior nodes, and consider the response at frequency $\omega$ of the resulting two terminal network. The only forces at nodes 3 and 4 are the inertial forces $\mathbf{f}_{3}=m \omega^{2} \mathbf{u}_{3}$ and $\mathbf{f}_{4}=m \omega^{2} \mathbf{u}_{4}$. Substituting this in (3) and eliminating $\mathbf{u}_{3}$ and $\mathbf{u}_{4}$ from the resulting equations, gives a relation between $\left(\mathbf{f}_{1}, \mathbf{f}_{2}\right)$ and $\left(\mathbf{u}_{1}, \mathbf{u}_{2}\right)$ exactly of the form (11) with a spring stiffness

$$
k(\omega)=\frac{2 g m \omega^{2}}{m \omega^{2}-2 g} .
$$

Thus this dispersive normal spring behaves exactly like a normal spring but with a frequency dependent spring stiffness, which will be negative for frequencies below $\omega_{0}=$ $\sqrt{2 g / m}$. The masses in the dispersive normal spring do not move, to first order in the displacement, when the two terminal nodes undergo a rigid body motion. They only move when the spring is extended or compressed.

The dispersive normal spring constructed here is floppy. For example, within the framework of linear elasticity, the interior diamond can be infinitesimally rotated with no change in the forces $\mathbf{f}_{1}, \mathbf{f}_{2}, \mathbf{f}_{3}$ and $\mathbf{f}_{4}$ and displacements $\mathbf{u}_{1}, \mathbf{u}_{2}, \mathbf{u}_{3}$ and $\mathbf{u}_{4}$ at the terminals. Strictly speaking one should perturb the network by adding a scaffolding of additional springs with very small spring constants to uniquely determine the interior displacements, but we refrain from doing so as to avoid complications. (Since then $\mathbf{f}_{1}$ will not be exactly equal to $-\mathbf{f}_{2}$.) The undetermined displacements do not effect the overall response of the dispersive normal spring. However associated with this degree of freedom is a buckling mode: when the springs in the network are under compression rather than tension one can expect, within the framework of non-linear elasticity, that the interior diamond will rotate, one way or the other, to relieve this compression. We ignore this since we are working only within the framework of linear elasticity.

We will now forget about the internal structure of the dispersive normal spring and treat it as a single object.

\section{A material having frequency dependent elasticity ten- sors and zero effective density}

Suppose we have have a periodic network (such as a triangular network) of identical normal springs with spring stiffness $k$. Let the nodes of this network be called "primary nodes". The effective elasticity tensor of the network $\mathbf{C}$ will be proportional to $k$ and we can write $\mathbf{C}=k \mathbf{C}^{0}$. Now if we replace each spring in the network by a dispersive normal spring with the same spring stiffness $k(\omega)$ given by (4), then the resulting material will have a frequency dependent effective elasticity tensor

$$
\mathbf{C}=k(\omega) \mathbf{C}^{0}=2 g m \omega^{2} \mathbf{C}^{0} /\left(m \omega^{2}-2 g\right),
$$

which is negative definite for frequencies below $\omega_{0}=\sqrt{2 g / m}$.

At the same time, within the framework of linear elasticity, the effective density of this network will be zero! By this we mean that at any fixed frequency, and to first order in the displacements, that the macroscopic response of the network will be the same as 


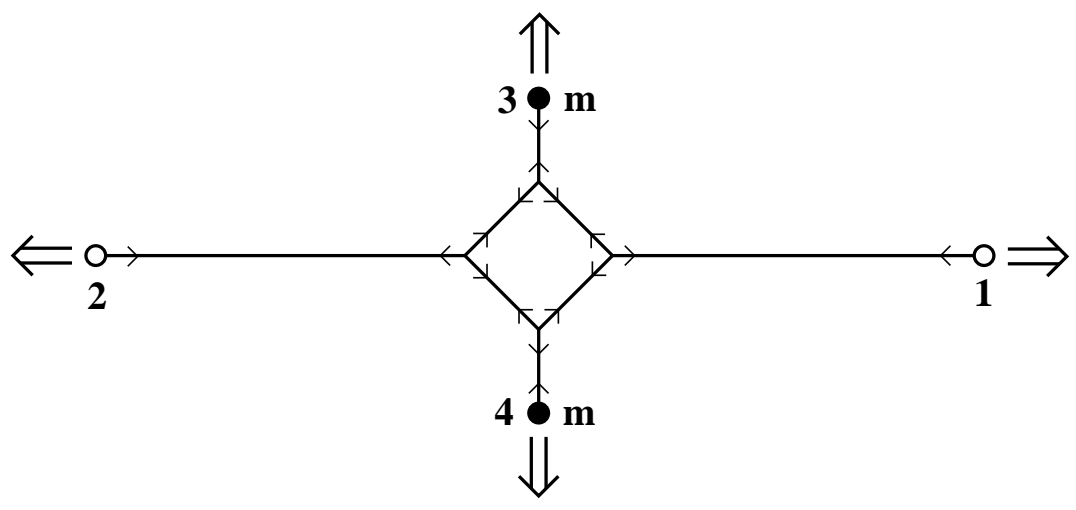

Figure 1: Sketch of the dispersive normal spring. The open circles represent terminal nodes, and the closed circles could be either terminal nodes or interior nodes with masses attached. The straight lines represent springs. The large arrows represent external or inertial forces acting on the nodes at one instant in time. The two small arrows on each spring give the direction of the force which the spring exerts on the node nearest to the arrow.

that of a periodic network of identical normal springs, without masses at the nodes. The internal masses cause the effective elasticity tensor to depend on frequency (and cause it to be negative definite for $\omega<\omega_{0}$ ) but do not contribute to the effective density, since the internal masses do not move (to first order in the displacements) when the primary node lattice is translated. Of course, as opposed to normal linear elasticity which can be useful even if there are large displacements such as rotations, the linear elasticity approximation here will only be valid for displacements which are small compared to the length of the springs in the network, if at all.

Some care needs to be applied in this notion of effective elasticity tensors. If we take a large sample of the periodic network of dispersive normal springs then we need to ensure that any cut dispersive normal spring at the boundary of the sample is removed and that there are no interior nodes of the dispersive normal springs in contact with the surface loadings applied to the boundary of the sample. If there are body forces (perhaps due to electric field gradients and polarizable nodes) then we need to make sure these only act on the primary nodes common to the original network of normal springs and not on the internal nodes and masses of each individual dispersive normal spring.

\section{Acknowledgements}

GWM is grateful for support from the University of Toulon-Var and from the National Science Foundation through grant DMS-0707978. 


\section{References}

[1] J. G. Berryman, Journal of the Acoustical Society of America 68(6), 1809-1819 (1980).

[2] P. Sheng, J. Mei, Z. Liu, and W. Wen, Physica. B, Condensed Matter 394, 256-261 (2007).

[3] M. Schoenberg and P. N. Sen, The Journal of the Acoustical Society of America 73(1), 61-67 (1983).

[4] G. Bouchitté and D. Felbacq, Comptes Rendus des Séances de l'Académie des Sciences. Série I. Mathématique 339, 377-382 (2004).

[5] Z. Liu, C. T. Chan, and P. Sheng, Physical Review B 71, 014103 (2005).

[6] A. Ávila, G. Griso, and B. Miara, Comptes Rendus des Séances de l'Académie des Sciences. Série I. Mathématique 340, 933-938 (2005).

[7] G. W. Milton, M. Briane, and J. R. Willis, New Journal of Physics 8, 248 (2006).

[8] G. W. Milton and J. R. Willis, Proc. R. Soc. A 463(2079), 855-880 (2007).

[9] F. G. Vasquez, G. W. Milton, and D. Onofrei, Journal of Elasticity(102), 31-54 (2011).

[10] M. Camar-Eddine and P. Seppecher, Archive for Rational Mechanics and Analysis 170(3), 211-245 (2003). 